\title{
Comparison of Sorafenib versus Hepatic Arterial Infusion Chemotherapy-Based Treatment for Advanced Hepatocellular Carcinoma with Portal Vein Tumor Thrombosis
}

\author{
Young Eun Ahn', Sang Jun Suh", Hyung Joon Yim¹, Yeon Seok Seo', Eileen L. Yoon², Tae Hyung Kim¹, \\ Young Sun Lee ${ }^{1}$, Sun Young Yim¹, Hae Rim Kim¹, Seong Hee Kang ${ }^{3}$, Young Kul Jung ${ }^{1}$, Ji Hoon Kim¹, \\ Jong Eun Yeon ${ }^{1}$, Soon Ho Um¹, and Kwan Soo Byun ${ }^{1}$ \\ ${ }^{1}$ Department of Internal Medicine, Korea University College of Medicine, ${ }^{2}$ Department of Internal Medicine, Inje University College of \\ Medicine, Seoul, and ${ }^{3}$ Department of Internal Medicine, Yonsei University Wonju College of Medicine, Wonju, Korea
}

\section{Article Info}

Received October 28, 2019

Revised February 8, 2020

Accepted February 21, 2020

Published online April 22, 2020

\section{Corresponding Author}

Hyung Joon Yim

ORCID https://orcid.org/0000-0002-6036-2754

E-mail gudwns21@korea.ac.kr

Yeon Seok Seo

ORCID https://orcid.org/0000-0003-4171-6331

E-mail drseo@korea.ac.kr

Young Eun Ahn and Sang Jun Suh contributed equally to this work as first authors.
Background/Aims: Sorafenib is the first approved systemic treatment for advanced hepatocellular carcinoma (HCC). However, its clinical utility is limited, especially in Asian countries. Several reports have suggested the survival benefits of hepatic arterial infusion chemotherapy (HAIC) for advanced HCC with main portal vein tumor thrombosis (PVTT). This study aimed to compare the efficacy of sorafenib-based therapy with that of HAIC-based therapy for advanced HCC with main PVTT.

Methods: Advanced HCC patients with main PVTT treated with sorafenib or HAIC between 2008 and 2016 at Korea University Medical Center were included. We evaluated overall survival (OS), time-to-progression (TTP), and the disease control rate (DCR).

Results: Seventy-three patients were treated with sorafenib $(n=35)$ or HAIC $(n=38)$. Baseline characteristics were not significantly different between groups, except the presence of solid organ metastasis $(46 \%$ vs $5.3 \%, p<0.001)$. The median OS time was not significantly different between the groups (6.4 months vs 10.0 months, $p=0.139)$. TTP was longer in the HAIC group than in the sorafenib group ( 2.1 months vs 6.2 months, $p=0.006)$. The DCR was also better in the HAIC group than in the sorafenib group $(37 \%$ vs $76 \%, p=0.001)$. Subgroup analysis, which excluded patients with extrahepatic solid organ metastasis, showed the same trends for the median OS time (8.8 months vs 11.1 months, $p=0.097)$, TTP (1.9 months vs 6.0 months, $p<0.001)$, and DCR $(53 \%$ vs $81 \%, p=0.030)$.

Conclusions: HAIC-based therapy may be an alternative to sorafenib for advanced $\mathrm{HCC}$ with main PVTT by providing longer TTP and a better DCR. (Gut Liver 2021;15:284-294)

Key Words: Carcinoma, hepatocellular; Portal vein thrombosis; Sorafenib; Hepatic artery; Chemotherapy

\section{INTRODUCTION}

Hepatocellular carcinoma (HCC) is a global health issue that presents with poor survival outcomes. ${ }^{1,2}$ Sorafenib is the firstly approved systemic treatment for HCC. ${ }^{3-5}$ It is indicated for patients with well-preserved liver function (Child-Pugh class A) and advanced tumors (i.e., Barcelona Clinic of Liver Cancer stage C). The survival benefits of sorafenib have been shown in two random- ized placebo-controlled trials. ${ }^{3,4}$ In the Sorafenib Hepatocellular Carcinoma Assessment Randomized Protocol (SHARP trial; a multicenter, phase III, double-blind, and placebo-controlled trial), 602 patients with advanced HCC in North America and Europe were enrolled. Sorafenib treatment increased the median overall survival (OS) from 7.9 months in the placebo group to 10.7 months in the sorafenib group (hazard ratio, 0.69), which represented a $31 \%$ decrease in the relative mortality risk. ${ }^{4}$ In a parallel 
phase III trial, which was conducted in the Asian-Pacific population, 226 patients with advanced HCC were enrolled in China, Taiwan, and South Korea. It also showed longer survival in the sorafenib group than in the placebo group (6.5 months vs 4.2 months; hazard ratio, 0.68 ). ${ }^{3}$ Although a direct comparison between the two studies requires caution, the worsened outcome of patients included in the Asia-Pacific trial compared with that in the SHARP trial may be attributed to patient characteristics. The AsiaPacific trial enrolled patients with more advanced disease (Eastern Cooperative Oncology Group [ECOG] 1-2 or metastatic disease) who were more likely to have worsened liver disease etiology, such as hepatitis B virus (HBV) infection. A global, non-intentional, surveillance study to evaluate the safety of sorafenib under real-life practice condition, the GIDEON (Global Investigation of Therapeutic Decisions in Hepatocellular Carcinoma and of Its Treatment with Sorafenib) study, also showed similar findings, suggesting more common worsened prognosis factors in Asian populations. ${ }^{6}$ In particular, as the geographical distribution of the main risk factors for HCC predominates as HBV in Asia (70\%) than in Europe (10\% to $15 \%)$ or North America (20\%), ${ }^{1,2}$ decreased efficacy of sorafenib is expected in patients of the Asia-Pacifica region.

Moreover, the previously mentioned two large phase III trials enrolled patients restricted mainly to ChildPugh class A; thus, sorafenib efficacy may decline in real practice if the range of patients is expanded to those with poor liver function. Indeed, many studies reported that the efficacy of sorafenib was reduced as the Child-Pugh score increased. ${ }^{7-11}$

Hepatic arterial infusion chemotherapy (HAIC) was developed in Japan and used for patients with advanced HCC in practice, although it is not preferred in Western countries because there are no data from randomized controlled trials. The theoretical advantage of HAIC is that the chemotherapeutic agent is more concentrated at the hepatic tumor and the adverse effects are milder than those observed with systemic chemotherapy. Currently several studies, which were conducted in Asian countries, suggested favorable outcomes of HAIC for HCC with portal vein tumor thrombosis (PVTT) ${ }^{12-16}$ In those studies, the median OS and time-to-progression (TTP) were estimated to be 7-9 months and 2-6 months, respectively, in HCC patients with PVTT who were treated with HAIC. ${ }^{12-16}$

A direct comparison between sorafenib and HAIC has not been well evaluated in a real-life setting. Importantly, the comparison of sorafenib and HAIC in a population with many chronic HBV infections and Child-Pugh class $B$ is necessary. If the efficacy is proved to be comparable between the two treatment modalities, HAIC may be useful in patients who cannot be treated with sorafenib because of costliness, decreased liver function, or adverse effects related to sorafenib. Thus, we aimed to compare the efficacy of sorafenib and HAIC in HCC patients with main PVTT to evaluate if HAIC can be an alternative option for advanced HCC treatment.

\section{MATERIALS AND METHODS}

\section{Patients' eligibility}

Between January 2008 and December 2016, 90 patients with advanced HCC were treated with sorafenib or HAIC at the Korea University Medical Center. Among them, we enrolled 73 HCC patients with main PVTT and retrospectively analyzed their clinical data. Main PVTT was defined as tumor thrombosis in the main portal trunk on radiographic images such as computed tomography or magnetic resonance images with dynamic enhance study. The study protocol was approved by the Institutional Review Board at Korea University (IRB number: AS13115) and conformed to the Declaration of Helsinki. The requirement for obtaining informed consent from patients was waived because of the retrospective nature of the study.

\section{Hepatic artery catheterization and chemoport insertion}

A catheter was inserted through the femoral artery using the Seldinger method. After localization of HCC, a 3-F heparin-coated catheter was inserted, and its tip was advanced to the common or proper hepatic artery. The other end of the catheter was connected to the chemoport, which was implanted in a subcutaneous pocket created in the inguinal region. The gastroduodenal and right gastric arteries were occluded with a coil to prevent gastroduodenal injury by chemotherapeutic agents.

\section{Treatment protocol}

Patients received repeated HAIC via the chemoport. At every cycle of chemotherapy, the tip of the catheter was ascertained by an interventional radiologist to prevent damage to other organs. HAIC was performed with an infusion of cisplatin $\left(60 \mathrm{mg} / \mathrm{m}^{2}\right)$ for 1 day and 5-flurouracil (500 $\mathrm{mg} / \mathrm{m}^{2}$ ) for 3 days every 4 weeks, as previously reported. ${ }^{17}$ Sorafenib was initiated with $400 \mathrm{mg}$ twice daily, and the dose was adjusted according to adverse events.

\section{Response and safety evaluation and definitions}

Treatment response was assessed according to the modified Response Evaluation Criteria in Solid Tumors. ${ }^{18}$ The disease control rate (DCR) was defined as the propor- 
tion of patients who had a best response rating of complete response, partial response, or stable disease, which was maintained for 4 weeks since the first manifestation of that rating. OS, which is the primary endpoint, was defined as the time from assignment of therapy for HCC with PVTT to death. ${ }^{19}$ Patients alive at the end of follow-up were censored. TTP was defined as the time from assignment of therapy for HCC with PVTT to radiological progression. ${ }^{18,19}$ Deaths during follow-up without evidence of radiological progression were censored. ${ }^{19}$ Safety and adverse events were assessed by reviewing medical records and laboratory findings according to the Common Terminology Criteria for Adverse Events v5.0 (https://ctep.cancer.gov/ protocolDevelopment/electronic_applications/ctc.htm). ${ }^{20}$

\section{Statistical analysis}

Statistical analysis was performed using the Statistical Package for the Social Sciences software version 18 (SPSS Inc., Chicago, IL, USA). Differences between the groups were examined for statistical significance using the Student $\mathrm{t}$-test or the chi-square test. The cumulative survival rate and tumor progression rate were calculated from the date of initiation of each therapy and assessed using the Kaplan-Meier life-table method, and differences were evalu- ated using the log-rank test. Tumor response to treatment was evaluated using the chi-square test. Univariate and multivariate analyses of factors that influenced survival were assessed using the Cox proportional hazard model. Statistical significance was defined as a two-sided p-value $<0.05$.

\section{RESULTS}

\section{Baseline characteristics}

A total of 73 patients were treated with sorafenib $(n=35)$ and HAIC $(n=38)$. The patients were older in the sorafenib group than in the HAIC group $(58.3 \pm 9.5$ years vs $53.0 \pm 11.6$ years, $\mathrm{p}=0.037$ ). The etiology of chronic liver disease was mostly chronic HBV infection, with no statistical difference between the groups $(\mathrm{p}=0.230)$. The proportion of patients with Child-Pugh class A liver function (69\% vs $71 \%, \mathrm{p}=0.817)$ and ECOG performance status $0-1$ ( $69 \%$ vs $77 \%, \mathrm{p}=0.892)$ was not different between the sorafenib and HAIC groups. About two-thirds of patients in each group were treated with sorafenib (60\%) or HAICbased $(76 \%)$ therapy as the first treatment and others were treated with radiofrequency ablation or resection before.

Table 1. Baseline Characteristics

\begin{tabular}{|c|c|c|c|}
\hline Characteristic & $\begin{array}{l}\text { Sorafenib-based } \\
\qquad(n=35)\end{array}$ & $\begin{array}{c}\text { HAIC-based } \\
(n=38)\end{array}$ & p-value \\
\hline Age, yr & $58.3 \pm 9.5$ & $53.0 \pm 11.6$ & 0.037 \\
\hline Male sex & $30(86)$ & 30 (79) & 0.450 \\
\hline \multicolumn{4}{|l|}{ Etiology } \\
\hline $\mathrm{CHB} / \mathrm{CHC} /$ alcohol/cryptogenic, $\mathrm{n}$ & $24 / 2 / 6 / 3$ & $33 / 2 / 2 / 1$ & 0.230 \\
\hline $\mathrm{CHB}$ or $\mathrm{CHC}+$ alcohol & $12(46)$ & $14(54)$ & 0.820 \\
\hline Child-Pugh class A/B & $24(69) / 11(31)$ & $27(71) / 11(29)$ & 0.817 \\
\hline Performance status by ECOG $0-1 / 2 / 3$ & $24(69) / 9(25) / 2(6)$ & $29(77) / 7(18) / 2(5)$ & 0.892 \\
\hline \multicolumn{4}{|l|}{ Prior treatment } \\
\hline None/curative (resection, RFA) & $21(60) / 14(40)$ & $29(76) / 9(24)$ & 0.134 \\
\hline Tumor type nodular/infiltrative & $16(46) / 19(54)$ & $14(37) / 24(63)$ & 0.441 \\
\hline LN metastasis & $14(40)$ & $13(34)$ & 0.609 \\
\hline Solid organ metastasis & $16(46)$ & $2(5.3)$ & $<0.001$ \\
\hline Lung/adrenal/bone/peritoneum, n & $12 / 2 / 1 / 1$ & $0 / 1 / 0 / 1$ & \\
\hline \multicolumn{4}{|l|}{ Laboratory findings } \\
\hline Platelet count, $\times 10^{3} / \mu \mathrm{L}$ & $196 \pm 105$ & $162 \pm 83$ & 0.133 \\
\hline Albumin, g/dL & $3.53 \pm 0.40$ & $3.48 \pm 0.43$ & 0.623 \\
\hline AST, IU/mL & $103 \pm 98$ & $89 \pm 63$ & 0.484 \\
\hline $\mathrm{ALT}, \mathrm{IU} / \mathrm{mL}$ & $56 \pm 47$ & $58 \pm 35$ & 0.835 \\
\hline Total bilirubin, mg/dL & $1.16 \pm 0.55$ & $1.04 \pm 0.48$ & 0.302 \\
\hline PT, INR & $1.16 \pm 0.12$ & $1.13 \pm 0.13$ & 0.270 \\
\hline AFP, ng/mL & $69,745 \pm 21,274$ & $71,341 \pm 14,823$ & 0.971 \\
\hline PIVKA-II, mAU/mL & $1,437 \pm 824$ & $1,361 \pm 821$ & 0.714 \\
\hline
\end{tabular}

Data are presented as mean \pm SD or number (\%).

HAIC, hepatic arterial infusion chemotherapy; CHB, chronic hepatitis B; CHC, chronic hepatitis C; ECOG, Eastern Cooperative Oncology Group; RFA, radiofrequency ablation; LN, lymph node; AST, aspartate aminotransferase; ALT, alanine aminotransferase; PT, prothrombin time; INR, international normalized ratio; AFP, alpha-fetoprotein; PIVKA-II, protein induced by vitamin K absence or antagonist-II. 
All patients were Barcelona Clinic of Liver Cancer stage $\mathrm{C}$ owing to portal vein invasion, and the PVTT status was Vp4 (presence of a tumor thrombus in the main trunk of the portal vein or a portal vein branch contralateral to the primarily involved lobe or both). The tumor status differed for solid organ metastasis. More patients with solid organ metastasis were treated with sorafenib than with HAIC ( $46 \%$ vs $5.3 \%, \mathrm{p}<0.001$ ). The proportions of infiltrative type (54\% vs $63 \%, \mathrm{p}=0.441)$ and lymph node metastasis $(40 \%$ vs $34 \%, \mathrm{p}=0.609$ ) were not different between the sorafenib and HAIC groups. The most common site of metastasis, in order, was the lung, adrenal gland, and bones. Laboratory findings were not different between the groups (Table 1).

\section{Treatment response and efficacy of chemotherapy}

The mean treatment durations were 2.8 (median, 2.0 months [range, 0.5 to 9.9 months]) and 3.9 (median, 2.8 months [range, 0.1 to 11.7 months]) months in the sorafenib and HAIC groups $(\mathrm{p}=0.120)$, respectively. In comparison with the sorafenib group, the median OS (6.4 months vs 10.0 months, $\mathrm{p}=0.139$ ) (Fig. $1 \mathrm{~A}$ ) tended to be longer in the HAIC group but there was no statistical difference. However, the TTP (2.1 months vs 6.2 months, $\mathrm{p}=0.006$ ) (Fig. $1 \mathrm{~B}$ ) was significantly longer in the HAIC group.

Final treatment responses such as progressive disease, stable disease, partial response, and complete response were noted in $28(80 \%)$, seven (20\%), 0 , and 0 patients of the sorafenib group, respectively, and 23 (60.5\%), 13 (34.2\%), one (2.6\%), and one (2.6\%) patient of the HAIC group, respectively $(\mathrm{p}=0.059)$ (Table 2$)$. One patient $\mathrm{ex}$ perienced complete response in the HAIC group and was
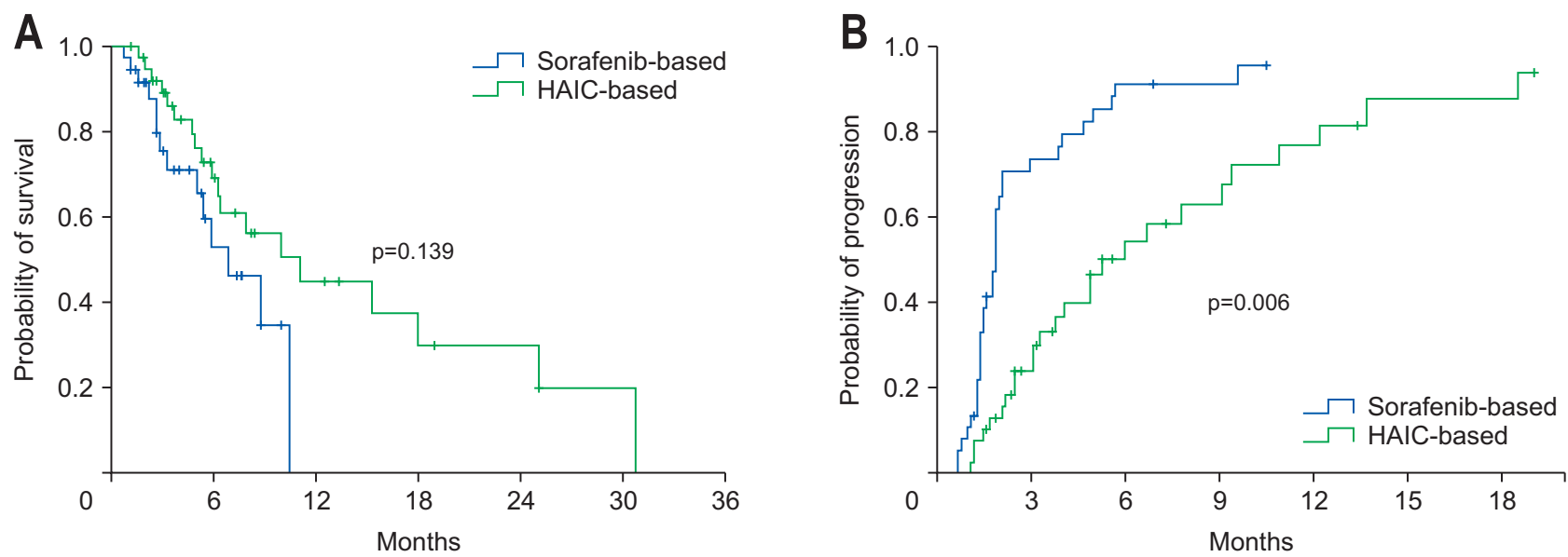

Fig. 1. Overall survival time and time-to-progression in the whole cohort. Comparison of survival (A) and time-to-progression (B) between the sorafenib and hepatic arterial infusion chemotherapy (HAIC) groups.

Table 2. Efficacy and Response Rate of Sorafenib and HAIC-Based Therapy

\begin{tabular}{|c|c|c|c|c|c|c|}
\hline \multirow{2}{*}{ Treatment response } & \multicolumn{3}{|c|}{ Overall patients $(n=73)$} & \multicolumn{3}{|c|}{ Patients without solid organ metastasis ( $n=55$ ) } \\
\hline & Sorafenib ( $n=35$ ) & HAIC (n=38) & p-value & Sorafenib (n=19) & HAIC $(n=36)$ & $\mathrm{p}$-value \\
\hline Overall survival, mo & & & 0.150 & & & 0.075 \\
\hline Median (range) & $6.4(0.8-31.0)$ & $10.0(1.2-30.8)$ & & $10.7(1.2-14.3)$ & $11.1(1.2-30.8)$ & \\
\hline $95 \% \mathrm{Cl}$ & $0.6-12.2$ & $5.9-14.1$ & & $4.2-17.2$ & $6.0-16.2$ & \\
\hline Time-to-progression, mo & & & 0.006 & & & 0.016 \\
\hline Median (range) & $2.1(0.7-10.7)$ & $6.2(0.2-30.8)$ & & $2.2(1.2-10.7)$ & $6.2(0.2-30.8)$ & \\
\hline $95 \% \mathrm{Cl}$ & $1.9-2.3$ & $3.6-8.8$ & & $1.8-2.6$ & $4.1-8.3$ & \\
\hline Level of response, No (\%) & & & 0.150 & & & 0.463 \\
\hline Complete response & 0 & $1(2.6)$ & & 0 & $1(2.8)$ & \\
\hline Partial response & 0 & $1(2.6)$ & & 0 & $1(2.8)$ & \\
\hline Stable disease & $7(20.0)$ & 13 (34.2) & & $4(21.1)$ & $13(36.1)$ & \\
\hline Progressive disease & $28(80.0)$ & $23(60.5)$ & & 15 (79.0) & 21 (58.3) & \\
\hline DCR, No. (\%)* & 13 (37.1) & 29 (76.3) & 0.001 & $10(52.6)$ & $29(80.6)$ & 0.030 \\
\hline
\end{tabular}

HAIC, hepatic arterial infusion chemotherapy; $\mathrm{Cl}$, confidence interval; DCR, disease control rate.

*DCR defined as the proportion of patients who had a "best response rating" of complete response, partial response, or stable disease, which was maintained for 4 weeks from the first manifestation of the rating. 
followed up for 16 months without recurrence. The best treatment responses such as above during chemotherapy was noted in 22,10 , three, and 0 patients of the sorafenib group, respectively, and nine, 22, six, and one patient of the HAIC group ( $\mathrm{p}=0.001$ ), respectively. DCRs were $37.1 \%$ and $76.3 \%$ in the sorafenib and HAIC groups, respectively $(\mathrm{p}=0.001)$, indicating better control in the HAIC group.

\section{Subgroup analysis}

\section{1) Patients without extrahepatic metastasis}

As there was a difference between the two groups in the proportion of extrahepatic solid organ metastasis, we performed a subgroup analysis excluding patients with solid organ metastasis. The median OS showed no statistical difference between the sorafenib and the HAIC groups ( 8.8 months vs 11.1 months, $\mathrm{p}=0.097$ ) (Fig. 2A). However, TTP was longer in the HAIC group (1.9 months vs 6.0 months, $\mathrm{p}<0.001$ ) (Fig. 2B). Likewise, compared with those treated with sorafenib, patients treated with HAIC showed better DCR ( $53 \%$ vs $81 \%, \mathrm{p}=0.030)$.

\section{2) Patients with or without combination therapy}

Details of the applied combination therapies are described in the next section. As the combination therapy might have influenced the results of the study, we have subdivided each treatment group into the monotherapy and combination therapy subgroups. The median OS was not significantly different among the four subgroups ( $\mathrm{p}=0.259$ ), whereas TTP showed significant differences among the four groups $(p=0.031)$. Post hoc analysis revealed that the median OS was not significantly different between the sorafenib monotherapy, sorafenib with combination therapy, HAIC monotherapy, and HAIC with combination therapy subgroups ( 10.7 months vs 5.1 months vs 15.3 months vs 10.0 months), except the median OS between the sorafenib with combination therapy and HAIC monotherapy subgroups ( $\mathrm{p}=0.032$ ) (Fig. 3A). The TTP was 2.1, $4.4,4.1$, and 6.2 months for the four subgroups, respectively, and that of the HAIC monotherapy $(\mathrm{p}=0.043)$ and HAIC with combination therapy subgroups $(\mathrm{p}=0.011)$ was significantly longer than that of the sorafenib monotherapy subgroup (Fig. 3B) by post hoc analysis.

The DCRs were $27.6 \%, 83.3 \%, 66.7 \%$, and $80.8 \%$ for the four subgroups, respectively $(\mathrm{p}<0.001)$.

\section{3) Patients with decreased liver function}

Twenty-two patients with Child-Pugh class B liver function were included. Fourteen patients had a Child-Turcotte-Pugh (CTP) score of 7 (seven in the sorafenib group and seven in the HAIC group), seven had a CTP score of 8 (four in the sorafenib group and three in the HAIC group), and one in the HAIC group had a CTP score of 9. The median OS of patients with a CTP score of 7 was 5.9 and 15.3 months $(\mathrm{p}=0.051)$ and that of patients with a CTP score of 8 or 9 was 3.4 and 5.9 months $(p=0.32$ ) in the sorafenib and HAIC groups, respectively. The TTP of patients with a CTP score of 7 was 2.0 and 3.9 months $(\mathrm{p}=0.332)$ and that of patients with a CTP score of 8 or 9 was 1.9 and 2.0 $(\mathrm{p}=0.308)$ in the sorafenib and HAIC groups, respectively. The DCRs of patients with a CTP score of 7 were $28 \%$ and $57 \%(\mathrm{p}=0.298)$ and those of patients with a CTP score of 8 or 9 were $0 \%$ and $75 \%(p=0.040)$ in the sorafenib and HAIC groups, respectively.

\section{4) Patients with poor performance status}

Four patients with an ECOG score of 3 were included in the study. Two belonged to the sorafenib group, and the other two to the HAIC group. Treatment was initiated only if improvement of performance status was possible or if the patient had a strong desire for anticancer therapy.
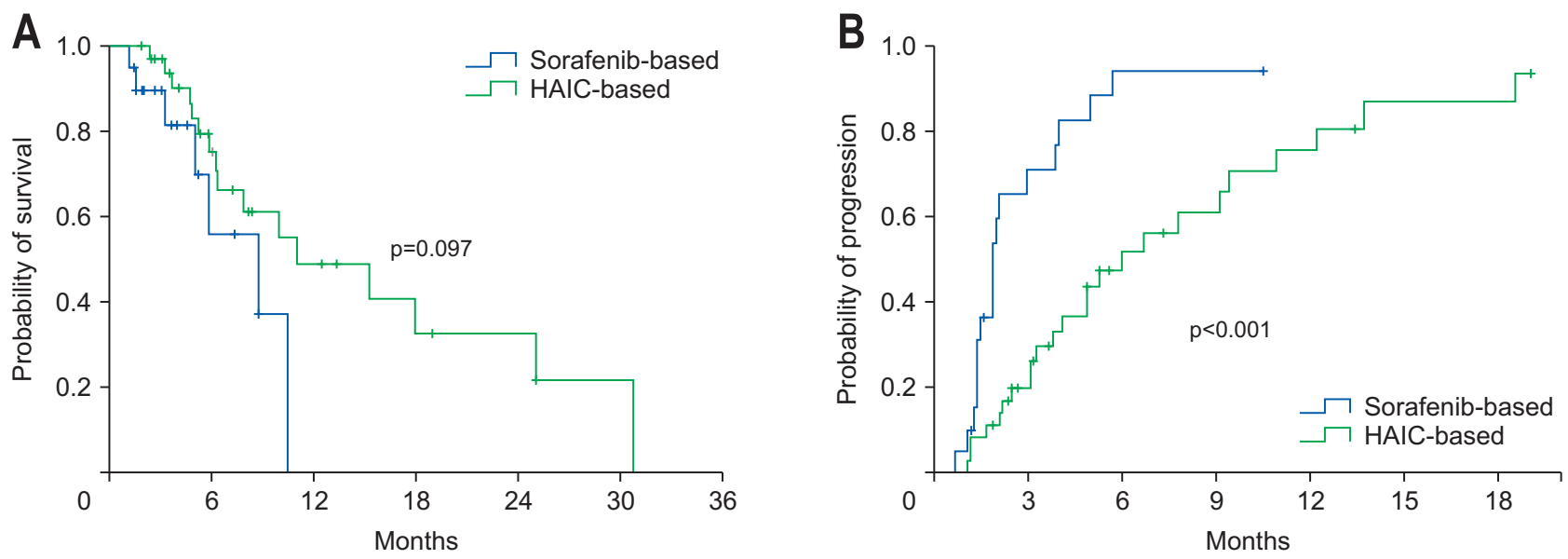

Fig. 2. Overall survival time and time-to-progression in patients without extrahepatic metastasis. Comparison of survival (A) and time-to-progression (B) between the sorafenib and hepatic arterial infusion chemotherapy (HAIC) groups. 
The median OS of patients with an ECOG score of 3 was 0.8 months in the sorafenib group and 6.3 months in the HAIC group ( $\mathrm{p}=0.317)$. The TTP of the patients with an ECOG score of 3 was 0.7 months in the sorafenib group and 4.1 months in the HAIC group ( $\mathrm{p}=0.09)$. In all patients with an ECOG score of 3, the final treatment response was progressive disease. The best treatment response during chemotherapy was a progressive disease for two patients in the sorafenib group and stable disease for two in the HAIC group. One of the patients in the sorafenib group reported grade 3 fatigue, nausea, and abdominal pain, and one of those in the HAIC group reported grade 4 gastrointestinal bleeding, grade 2 anemia, and grade 2 aspartate amino- transferase elevation. Another patient in the HAIC group reported spontaneous bacterial peritonitis after the second treatment cycle.

The detailed courses of the individual patients are as follows: one patient in the sorafenib group received medication for 20 days with a strong hope of improvement by treatment but died of pulmonary complications. The other patient in the sorafenib group was a chronic alcoholic whose performance had been impaired by alcohol drinking. The general condition of the patient showed a trend of improvement after abstinence, so we started treatment for HCC. The treatment was discontinued due to disease progression after 70 days. The third patient was diagnosed
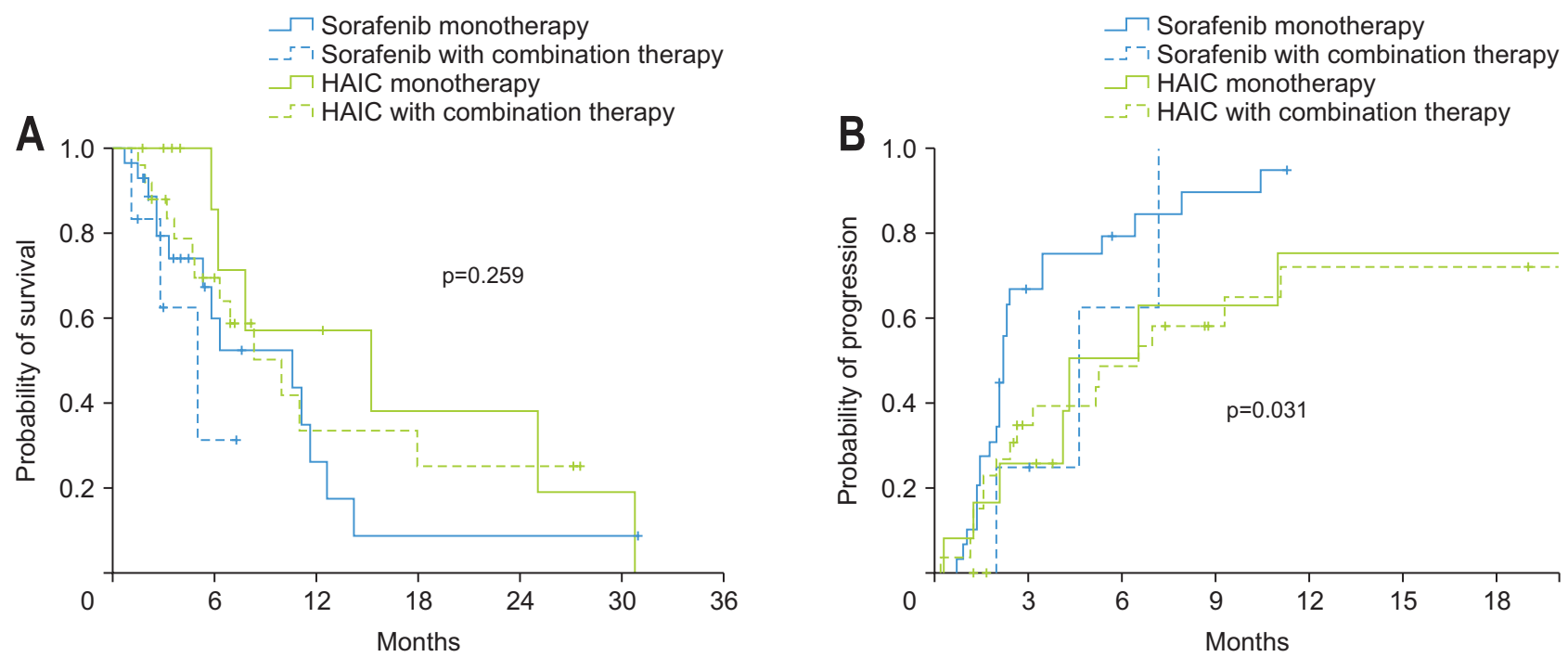

Fig. 3. Overall survival time and time-to-progression in the subgroup analysis. Comparison of survival (A) and time-to-progression (B) between the sorafenib monotherapy group, sorafenib with combination therapy group, hepatic arterial infusion chemotherapy (HAIC) monotherapy group and HAIC with combination therapy group.

Table 3. Multivariable Analysis for Overall Survival and Tumor Progression

\begin{tabular}{|c|c|c|c|c|c|c|}
\hline \multirow[t]{2}{*}{ Variable } & \multicolumn{3}{|c|}{$\begin{array}{l}\text { Factors associated with } \\
\text { overall survival }\end{array}$} & \multicolumn{3}{|c|}{$\begin{array}{l}\text { Factors associated with } \\
\text { tumor progression }\end{array}$} \\
\hline & $\mathrm{HR}$ & $95 \% \mathrm{Cl}$ & $\mathrm{p}$-value & $H R$ & $95 \% \mathrm{Cl}$ & $\mathrm{p}$-value \\
\hline Age & 0.994 & $0.960-1.029$ & 0.735 & 1.026 & $0.995-1.058$ & 0.105 \\
\hline Gender & 0.961 & $0.368-2.508$ & 0.968 & 0.956 & $0.407-2.339$ & 0.956 \\
\hline Child-Pugh class & 1.958 & $0.227-16.882$ & 0.541 & 1.725 & $0.577-5.156$ & 0.329 \\
\hline Performance status by ECOG & 1.341 & $0.311-5.784$ & 0.694 & 0.892 & $0.195-4.082$ & 0.883 \\
\hline Prior treatment & 0.655 & $0.413-1.038$ & 0.072 & 0.994 & $0.722-1.367$ & 0.969 \\
\hline Infiltrative tumor & 1.024 & $0.468-2.241$ & 0.953 & 1.009 & $0.541-1.882$ & 0.978 \\
\hline Lymph node metastasis & 1.050 & $0.437-2.520$ & 0.913 & 0.805 & $0.399-1.626$ & 0.546 \\
\hline Solid organ metastasis & 0.804 & $0.864-10.188$ & 0.084 & 1.534 & $0.672-3.501$ & 0.310 \\
\hline Current treatment* & 0.470 & $0.172-1.286$ & 0.141 & 0.571 & $0.260-1.252$ & 0.162 \\
\hline AFP $>200 \mathrm{ng} / \mathrm{mL}$ & 1.648 & $0.724-3.752$ & 0.234 & 1.936 & $0.948-3.956$ & 0.070 \\
\hline Combination therapy & 2.212 & $0.821-5.963$ & 0.117 & 0.972 & $0.457-2.068$ & 0.941 \\
\hline
\end{tabular}

$\mathrm{HR}$, hazard ratio; $\mathrm{Cl}$, confidence interval; ECOG, Eastern Cooperative Oncology Group; AFP, alpha-fetoprotein; HAIC, hepatic arterial infusion chemotherapy.

*Sorafenib-based or HAIC-based therapy. 
with HCC after tumor rupture, and started HAIC after emergent hepatic artery embolization. His initially poor performance status improved during treatment as there was no more bleeding event. The treatment was stopped due to the occurrence of lung metastasis after five cycles of HAIC for 124 days. Two months after treatment discontinuation, the patient died of hepatorenal syndrome. The remaining patient was eager to be treated and was started on HAIC treatment with $25 \%$ dose reduction. The patient received seven cycles of therapy for 187 days, but the treatment was discontinued owing to disease progression. $\mathrm{He}$ died of gastrointestinal bleeding 1 month after treatment discontinuation.

\section{Factors related to survival and tumor progression}

Using Cox's proportional hazard model, we investigated the factors related to survival. We evaluated if the treatment modality and solid organ metastasis affected survival. However, no factor was related to survival in the univariate and multivariate analyses of our study population (Table 3).

We also investigated factors related to tumor progression. Solid organ metastasis and treatment group were significantly related to tumor progression in the univariate analysis. However, no factor was associated with tumor

Table 4. Adverse Events Related to Treatment

\begin{tabular}{|c|c|c|c|c|}
\hline \multirow{2}{*}{ Adverse events } & \multicolumn{2}{|c|}{ Sorafenib-based ( $n=35)$} & \multicolumn{2}{|c|}{ HAIC-based ( $n=38$ ) } \\
\hline & All grades & Grade $3 \& 4$ & All grades & Grade $3 \& 4$ \\
\hline \multicolumn{5}{|l|}{ Hematologic } \\
\hline Neutropenia & $1(2.9)$ & $1(2.9)$ & 9 (23.7) & $4(10.5)$ \\
\hline Anemia & $7(20.0)$ & $1(2.9)$ & 14 (36.8) & $3(7.9)$ \\
\hline Thrombocytopenia & $3(8.6)$ & 0 & $17(44.7)$ & $8(21.1)$ \\
\hline \multicolumn{5}{|l|}{ Dermatologic } \\
\hline Hand-foot skin reaction & $10(28.6)$ & $3(8.6)$ & 0 & 0 \\
\hline Rash & $1(2.9)$ & 0 & 0 & 0 \\
\hline Scrotal erythema & $1(2.9)$ & 0 & 0 & 0 \\
\hline \multicolumn{5}{|l|}{ Gastrointestinal } \\
\hline Dyspepsia/anorexia & $9(25.7)$ & $2(5.7)$ & $5(13.2)$ & $1(2.6)$ \\
\hline Nausea/vomiting & $7(20.0)$ & $3(8.6)$ & $4(10.5)$ & 0 \\
\hline Diarrhea/constipation & $6(17.1)$ & $2(5.7)$ & 2 (5.3) & $1(2.6)$ \\
\hline Abdominal pain & $3(8.6)$ & $1(2.9)$ & $2(5.3)$ & 0 \\
\hline GI bleeding & $2(5.7)$ & $1(2.9)$ & $1(2.6)$ & $1(2.6)$ \\
\hline HCC rupture & $2(5.7)$ & $2(5.7)$ & $1(2.6)$ & $1(2.6)$ \\
\hline \multicolumn{5}{|l|}{ Constitutional } \\
\hline Fatigue & $10(28.6)$ & $5(14.3)$ & $3(7.9)$ & 0 \\
\hline General weakness & $2(5.7)$ & 0 & $3(7.9)$ & $2(5.3)$ \\
\hline \multicolumn{5}{|l|}{ Laboratory } \\
\hline AST elevation & $8(22.9)$ & $8(22.9)$ & $6(15.8)$ & $5(13.2)$ \\
\hline ALT elevation & $2(5.7)$ & $1(2.9)$ & 5 (13.2) & $3(7.9)$ \\
\hline Bilirubin elevation & $13(37.1)$ & $6(17.1)$ & $6(15.8)$ & 0 \\
\hline PT INR elevation & $12(34.3)$ & 0 & $6(15.8)$ & 0 \\
\hline Hyponatremia & $1(2.9)$ & $1(2.9)$ & 0 & 0 \\
\hline \multicolumn{5}{|l|}{ Complication of liver cirrhosis } \\
\hline Ascites aggravation & $2(5.7)$ & $2(5.7)$ & 0 & 0 \\
\hline SBP & $1(2.9)$ & $1(2.9)$ & $2(5.3)$ & $2(5.3)$ \\
\hline Hepatic encephalopathy & $2(5.7)$ & $2(5.7)$ & $1(2.6)$ & $1(2.6)$ \\
\hline \multicolumn{5}{|l|}{ Catheter related complication } \\
\hline Catheter obstruction & 0 & 0 & $2(5.3)$ & $1(2.6)$ \\
\hline Chemoport site infection & 0 & 0 & $4(10.5)$ & $3(7.9)$ \\
\hline Hepatic artery total occlusion & 0 & 0 & $1(2.6)$ & $1(2.6)$ \\
\hline \multicolumn{5}{|l|}{ Others } \\
\hline Infection & 0 & 0 & $4(10.5)$ & $4(10.5)$ \\
\hline Febrile neutropenia & 0 & 0 & $1(2.6)$ & $1(2.6)$ \\
\hline Peripheral neuropathy & $1(2.9)$ & 0 & $1(2.6)$ & 0 \\
\hline Dizziness & $2(5.7)$ & 0 & $1(2.6)$ & 0 \\
\hline
\end{tabular}

Data are presented as number (\%).

HAIC, hepatic arterial infusion chemotherapy; GI, gastrointestinal; HCC, hepatocellular carcinoma; AST, aspartate aminotransferase; ALT, alanine aminotransferase; PT, prothrombin time; INR, international normalized ratio; SBP, spontaneous bacterial peritonitis. 
progression after correcting for other factors such as age and gender (Table 3).

\section{Safety and adverse events}

More patients experienced hematologic adverse events (anemia and thrombocytopenia) in the HAIC group than in the sorafenib group. Dermatologic adverse events (hand-foot syndrome and scrotal erythema) occurred in the sorafenib group. Lethal side effects, such as gastrointestinal bleeding (two patients in the sorafenib group and one patient in the HAIC group) and HCC rupture (two patients in the sorafenib group and one patient in the HAIC group), occurred in both the groups. More constitutional complications occurred in the sorafenib group than in the HAIC group. Catheter-related complications (catheter obstruction and chemoport site infection) occurred in the HAIC group, and appropriate treatments, such as reperfusion and antibiotics, have been undertaken for these patients (Table 4). Adverse events according to the CTP score are described separately in Supplementary Tables 1 and 2.

The main reason for treatment discontinuation was disease progression (17 patients in the sorafenib group vs nine patients in the HAIC group). The other reasons for treatment discontinuation, in the order of frequency, were drug side effects (three patients vs nine patients), chemoport complication (0 patient vs five patients), complications of liver cirrhosis (five patients vs four patients), tumor complication (four patients vs three patients), infection (0 patient vs one patient), hepatic dysfunction (three patients vs three patients), deterioration of general condition (two patients vs two patients), and transfer to other hospitals (one patient vs two patients).

\section{Combined therapy and second-line therapy}

In the sorafenib group, 29 patients $(83 \%)$ received sorafenib monotherapy, while six (17.1\%) received combination therapy during sorafenib treatment: two patients (5.7\%) received treatment combined with transarterial chemotherapy with lipiodol (TACL) and four (11.4\%) received treatment combined with 3-dimensional conformal radiotherapy. More patients received combination therapy in the HAIC group. Twelve patients (31.6\%) received HAIC monotherapy, while 26 patients $(68 \%)$ received combined therapy: six patients $(15.8 \%)$ received treatment combined with TACL, 10 (26.3\%) received treatment combined with radiotherapy, nine $(23.7 \%)$ received treatment combined with TACL and radiotherapy, and one (2.6\%) received treatment combined with radiotherapy and percutaneous ethanol injection therapy on PVTT.

In the sorafenib group, 28 patients (80\%) did not receive second-line therapy after cessation of sorafenib, while seven patients (20\%) received second-line therapy: systemic cytotoxic chemotherapy $(\mathrm{n}=6 ; 16.9 \%)$ and TACL $(\mathrm{n}=1 ; 2.9 \%)$. In the HAIC group, 31 patients (82\%) did not receive second-line therapy, while seven patients (18.4\%) received second-line therapy: systemic cytotoxic chemotherapy $(n=1 ; 2.6 \%)$, TACL $(n=4 ; 11 \%)$, and sorafenib $(\mathrm{n}=2 ; 5.3 \%)$.

\section{DISCUSSION}

The prognosis of patients with advanced HCC is poor, especially if there is a main PVTT, which is found in $10 \%$ to $40 \%$ of patients first diagnosed with $\mathrm{HCC}^{21-23}$ In patients with PVTT, an extrahepatic metastasis is often detected at diagnosis, resulting in fewer treatment options. ${ }^{21-23} \mathrm{OS}$ is shorter in HCC patients with PVTT than in those without PVTT. Previous studies have shown that OS is only 2 to 4 months with supportive care in HCC patients with PVTT, which is much shorter than the 10 to 24 months in HCC patients without PVTT. ${ }^{22-24}$ According to the Barcelona Clinic of Liver Cancer staging system, HCC with PVTT is classified as an advanced stage, and sorafenib is recommended as the first-line option. ${ }^{1}$ However, survival advantages were not high when sorafenib was administered; the OS was 6 to 10 months. ${ }^{3,4}$ In addition, the initial results of the study were mainly targeted to patients with favorable residual liver function (Child-Pugh class A) and good performance status (ECOG 0-1). Therefore, sorafenib is less effective in real practice. ${ }^{25}$

In the presence of a main PVTT, tumors in the liver are often progressed and intrahepatic metastasis to the opposite lobe can occur quickly. ${ }^{26}$ Hence, it is important to control tumors in the liver. HAIC is designed to reduce the systemic side effects of chemotherapy agents and to maximize tumor control by administering high concentrations of anticancer drugs to hepatic tumors. Although HCC does not respond well to conventional anticancer drugs, the infusion of relatively large amounts of chemotherapeutic agents will enhance anti-tumor responses, even in patients with marginal liver function, and avoid ischemic complications of transarterial therapy because it does not require hepatic arterial embolization. ${ }^{16,27,28}$ However, more data on these HCC patients with PVTT may be required. In addition, only a few studies have directly compared the efficacy of sorafenib and HAIC. ${ }^{29,30}$ Therefore, this study compared the effects of sorafenib and HAIC in patients with similar liver function and performance status.

A total of 73 patients were retrospectively examined. Chronic HBV infection was the most common cause of underlying liver disease. The proportion of Child-Pugh 
class B patients was one-third, which suggests that more patients with decreased liver function were included compared with that in previous studies. The proportion of patients with ECOG 2 or 3 was also one-third. Owing to the retrospective design, there was a difference between the two treatment groups. Solid organ metastasis was more common in the sorafenib group than in the HAIC group. Therefore, we performed subgroup analysis excluding patients with extrahepatic metastasis, and the results were consistent.

The final therapeutic response was mostly progressive disease, which agrees with findings of previous studies. ${ }^{3,6,12-15}$ In the HAIC group, one complete response was achieved, although the number was negligible. DCR was compared between the groups according to the best response during the treatment period, and the results were better in the HAIC group than in the sorafenib group. The median OS was 6.9 months in the sorafenib group and 11.1 months in the HAIC group, but there was no significant difference between groups, although patients in the HAIC group tended to have longer OS. However, the median TTP was significantly longer in the HAIC group than in the sorafenib group. In the additional subgroup analyses, a trend toward favoring HAIC therapy was observed in patients with Child-Pugh class B in terms of OS and TTP, albeit statistical significances were not demonstrated. HAIC was relatively tolerable in two patients with an ECOG performance status of 3 .

Although patients treated with HAIC-based therapy experienced more hematologic toxicity, the complications were mostly manageable. Sorafenib-based therapy was more often related to dermatologic toxicity (34\%, all grades) and thus required dose adjustment.

As this study was performed based on real practice, we acknowledge that patients in the HAIC group had more chance of receiving combined treatment such as radiotherapy. According to the medical insurance reimburse guidelines in Korea, sorafenib has not been allowed to combine other locoregional therapies. This situation may have influenced the outcomes regarding objective tumor responses. However, in the further sub-analysis, no significant differences in OS and TTP were found between the monotherapy and combination therapy when the sorafenib monotherapy and sorafenib-based combination therapy groups and the HAIC monotherapy and HAIC-based combination therapy groups were compared. Therefore, further research is needed to determine the strategy for combination therapy for advanced HCC.

However, even considering these factors, HAIC-based therapy should be an alternative to sorafenib therapy in patients who cannot tolerate or afford sorafenib because
HAIC-based treatment is 5 to 10 times less costly.

Currently, there are newer treatment options for advanced HCC patients, such as lenvatinib, which demonstrated non-inferior efficacy to sorafenib. ${ }^{31}$ Furthermore, second-line therapies are available using new molecular targeting therapies including regorafenib and cabozantinib, which showed survival benefits after sorafenib failure. ${ }^{32,33}$ However, most of these drugs were tested in patients with well-reserved liver function and good performance status, similar to the sorafenib trials. Hence, these drugs should also have limited applicability in real practice. Thus, we need to consider the utility of HAIC treatment even in the era of molecular target therapy. However, because the patients in this study did not receive second-line therapy with these new agents, further investigation is necessary.

This study had several limitations, including the nonrandomized, retrospective design and relatively small number of patients. However, we found that advanced HCC patients with or without extrahepatic metastases showed better DCR and longer TTP with HAIC than with sorafenib treatment. Furthermore, OS tended to be longer in the HAIC group than in the sorafenib group, although statistical significance was not observed. Another limitation is the non-inclusion of newer therapies as second-line therapy. Unfortunately, these therapies were not available in Korea during the study period.

In conclusion, HAIC-based therapy may be an alternative treatment option to sorafenib for advanced HCC with main portal vein thrombosis and may result in favorable DCR in patients with or without solid organ metastasis.

\section{CONFLICTS OF INTEREST}

No potential conflict of interest relevant to this article was reported.

\section{ACKNOWLEDGEMENTS}

This project was funded by the Korea University Research Grant.

The authors are grateful to Prof. Jaehyung Cha at Department of Biostatistics, Korea University for analyzing the data set.

\section{AUTHOR CONTRIBUTIONS}

Study design: H.J.Y. Data acquisition: E.L.Y., T.H.K., Y.S.L., S.Y.Y., H.R.K., S.H.K., Y.K.J., J.H.K., J.E.Y., S.H.U., 
K.S.B., H.J.Y., Y.S.S. Data analysis: Y.E.A., S.J.S. Funding: H.J.Y. Drafting of the manuscript: Y.E.A., S.J.S., H.J.Y. Reviewing the manuscript: E.L.Y., T.H.K., Y.S.L., S.Y.Y., H.R.K., S.H.K., Y.K.J., J.H.K., J.E.Y., S.H.U., K.S.B., H.J.Y. Critical revision of the manuscript: Y.S.S. Overall project management: H.J.Y. Approval of final manuscript: all authors.

\section{ORCID}

Young Eun Ahn https://orcid.org/0000-0001-5414-5772

Sang Jun Suh https://orcid.org/0000-0003-4128-3732

Hyung Joon Yim https://orcid.org/0000-0002-6036-2754

Yeon Seok Seo https://orcid.org/0000-0003-4171-6331

Eileen L. Yoon https://orcid.org/0000-0003-0474-048X

Tae Hyung Kim https://orcid.org/0000-0002-7747-4293

Young Sun Lee https://orcid.org/0000-0001-6396-0859

Sun Young Yim https://orcid.org/0000-0001-7346-5974

Hae Rim Kim https://orcid.org/0000-0002-4305-0839

Seong Hee Kang https://orcid.org/0000-0002-0783-509X

Young Kul Jung https://orcid.org/0000-0002-6566-1382

Ji Hoon Kim https://orcid.org/0000-0003-3924-0434

Jong Eun Yeon https://orcid.org/0000-0002-0510-7371

Soon Ho Um Thtps:/orcid.org/0000-0002-4545-7907

Kwan Soo Byun https://orcid.org/0000-0003-4031-842X

\section{REFERENCES}

1. European Association for the Study of the Liver. EASL Clinical Practice Guidelines: management of hepatocellular carcinoma. J Hepatol 2018;69:182-236.

2. Global Burden of Disease Liver Cancer Collaboration, Akinyemiju T, Abera S, et al. The burden of primary liver cancer and underlying etiologies from 1990 to 2015 at the global, regional, and national level: results from the global burden of disease study 2015. JAMA Oncol 2017;3:16831691.

3. Cheng AL, Kang YK, Chen Z, et al. Efficacy and safety of sorafenib in patients in the Asia-Pacific region with advanced hepatocellular carcinoma: a phase III randomised, double-blind, placebo-controlled trial. Lancet Oncol 2009;10:25-34.

4. Llovet JM, Ricci S, Mazzaferro V, et al. Sorafenib in advanced hepatocellular carcinoma. N Engl J Med 2008;359:378-390.

5. Yim HJ, Suh SJ, Um SH. Current management of hepatocellular carcinoma: an Eastern perspective. World J Gastroenterol 2015;21:3826-3842.

6. Lencioni R, Kudo M, Ye SL, et al. First interim analysis of the GIDEON (Global Investigation of therapeutic deci- sions in hepatocellular carcinoma and of its treatment with sorafeNib) non-interventional study. Int J Clin Pract 2012;66:675-683.

7. Kim HY, Park JW, Joo J, et al. Worse outcome of sorafenib therapy associated with ascites and Child-Pugh score in advanced hepatocellular carcinoma. J Gastroenterol Hepatol 2013;28:1756-1761.

8. Kudo M, Ueshima K, Arizumi T. Real-life clinical practice with sorafenib in advanced hepatocellular carcinoma: a single-center experience. Dig Dis 2012;30:609-616.

9. Pressiani T, Boni C, Rimassa L, et al. Sorafenib in patients with Child-Pugh class A and B advanced hepatocellular carcinoma: a prospective feasibility analysis. Ann Oncol 2013;24:406-411.

10. Tsukui Y, Mochizuki H, Hoshino Y, et al. Factors contributing to the overall survival in patients with hepatocellular carcinoma treated by sorafenib. Hepatogastroenterology 2012;59:2536-2539.

11. Woo HY, Heo J, Yoon KT, et al. Clinical course of sorafenib treatment in patients with hepatocellular carcinoma. Scand J Gastroenterol 2012;47:809-819.

12. Ikeda M, Okusaka T, Furuse J, et al. A multi-institutional phase II trial of hepatic arterial infusion chemotherapy with cisplatin for advanced hepatocellular carcinoma with portal vein tumor thrombosis. Cancer Chemother Pharmacol 2013;72:463-470.

13. Jeong SW, Jang JY, Lee JE, et al. The efficacy of hepatic arterial infusion chemotherapy as an alternative to sorafenib in advanced hepatocellular carcinoma. Asia Pac J Clin Oncol 2012;8:164-171.

14. Kim BK, Park JY, Choi HJ, et al. Long-term clinical outcomes of hepatic arterial infusion chemotherapy with cisplatin with or without 5-fluorouracil in locally advanced hepatocellular carcinoma. J Cancer Res Clin Oncol 2011;137:659-667.

15. Miyaki D, Aikata H, Honda $Y$, et al. Hepatic arterial infusion chemotherapy for advanced hepatocellular carcinoma according to Child-Pugh classification. J Gastroenterol Hepatol 2012;27:1850-1857.

16. Song DS, Bae SH, Song MJ, et al. Hepatic arterial infusion chemotherapy in hepatocellular carcinoma with portal vein tumor thrombosis. World J Gastroenterol 2013;19:46794688 .

17. Woo HY, Bae SH, Park JY, et al. A randomized comparative study of high-dose and low-dose hepatic arterial infusion chemotherapy for intractable, advanced hepatocellular carcinoma. Cancer Chemother Pharmacol 2010;65:373-382.

18. Lencioni R, Llovet JM. Modified RECIST (mRECIST) assessment for hepatocellular carcinoma. Semin Liver Dis 2010;30:52-60.

19. Llovet JM, Di Bisceglie AM, Bruix J, et al. Design and endpoints of clinical trials in hepatocellular carcinoma. J Natl 
Cancer Inst 2008;100:698-711.

20. National Cancer Institute. Common Terminology Criteria for Adverse Events v5.0 [Internet]. Bethesda: Cancer Therapy Evaluation Program [cited 2020 Mar 26]. Available from: https://ctep.cancer.gov/protocolDevelopment/electronic_ applications/ctc.htm.

21. Cheung TK, Lai CL, Wong BC, Fung J, Yuen MF. Clinical features, biochemical parameters, and virological profiles of patients with hepatocellular carcinoma in Hong Kong. Aliment Pharmacol Ther 2006;24:573-583.

22. Llovet JM, Bustamante J, Castells A, et al. Natural history of untreated nonsurgical hepatocellular carcinoma: rationale for the design and evaluation of therapeutic trials. Hepatology 1999;29:62-67.

23. Minagawa M, Makuuchi M. Treatment of hepatocellular carcinoma accompanied by portal vein tumor thrombus. World J Gastroenterol 2006;12:7561-7567.

24. Schöniger-Hekele M, Müller C, Kutilek M, Oesterreicher C, Ferenci P, Gangl A. Hepatocellular carcinoma in Central Europe: prognostic features and survival. Gut 2001;48:103-109.

25. Peck-Radosavljevic M, Greten TF, Lammer J, et al. Consensus on the current use of sorafenib for the treatment of hepatocellular carcinoma. Eur J Gastroenterol Hepatol 2010;22:391-398.

26. Fan J, Wu ZQ, Tang ZY, et al. Multimodality treatment in hepatocellular carcinoma patients with tumor thrombi in portal vein. World J Gastroenterol 2001;7:28-32.

27. Oh MJ, Lee HJ, Lee SH. Efficacy and safety of hepatic arte- rial infusion chemotherapy for advanced hepatocellular carcinoma as first-line therapy. Clin Mol Hepatol 2013;19:288299.

28. Woo HY, Heo J. New perspectives on the management of hepatocellular carcinoma with portal vein thrombosis. Clin Mol Hepatol 2015;21:115-121.

29. Choi JH, Chung WJ, Bae SH, et al. Randomized, prospective, comparative study on the effects and safety of sorafenib vs. hepatic arterial infusion chemotherapy in patients with advanced hepatocellular carcinoma with portal vein tumor thrombosis. Cancer Chemother Pharmacol 2018;82:469478.

30. Song DS, Song MJ, Bae SH, et al. A comparative study between sorafenib and hepatic arterial infusion chemotherapy for advanced hepatocellular carcinoma with portal vein tumor thrombosis. J Gastroenterol 2015;50:445-454.

31. Kudo M, Finn RS, Qin S, et al. Lenvatinib versus sorafenib in first-line treatment of patients with unresectable hepatocellular carcinoma: a randomised phase 3 non-inferiority trial. Lancet 2018;391:1163-1173.

32. Abou-Alfa GK, Meyer T, Cheng AL, et al. Cabozantinib in patients with advanced and progressing hepatocellular carcinoma. N Engl J Med 2018;379:54-63.

33. Bruix J, Qin S, Merle P, Granito A, et al. Regorafenib for patients with hepatocellular carcinoma who progressed on sorafenib treatment (RESORCE): a randomised, double-blind, placebo-controlled, phase 3 trial. Lancet 2017;389:56-66. 